\title{
Authors:
}

\section{6 - Loneliness and mortality in older adults and the role of depression}

N.Schutter (Arkin Menthal Health Care, Department of Geriatric Psychiatry, the Netherlands), T.J. Holwerda (Arkin Mental Health Care, Department of Psychiatry), D. Rhebergen (GGZ InGeest, Department of Psychiatry, Amsterdam Public Health Research Institute, VU University Medical Center), H.C. Comijs (GGZ InGeest, Department of Psychiatry, Amsterdam Public Health Research Institute, VU University Medical Center), J.J.M. Dekker (Arkin Mental Health Care, Department of Clinical Research, VU University Medical Center Amsterdam), M.L.Stek (GGZ InGeest, Department of Psychiatry, Amsterdam Public Health Research Institute, VU University Medical Center Amsterdam, the Netherlands).

\section{Background:}

The prevalence of loneliness increases with age. The presence of loneliness in older adults has been found to be associated with health problems such as depression, decreased cognitive functioning, increases in systolic blood pressure and increased mortality. The underlying mechanisms of the higher mortality risk are largely unknown.

\section{Methods:}

Meta-analysis to investigate the present evidence for the associations between loneliness and mortality. Cross-sectional studies investigating the associations between loneliness and cardiovascular disease and between loneliness and cortisol in 378 depressed and 132 non-depressed older adults.

\section{Results:}

Loneliness appears to be associated with increased mortality, although when only studies are included that consider depression as a covariate, the association is not significant. Therefore it seems likely that depression plays a mediating role in the higher mortality risk.

We did not find a significant association between loneliness and cardiovascular disease. In contrast, loneliness was significantly associated with lower cortisol output and decreased dexamethasone suppression.

\section{Discussion:}

The results and their implications for prevention and treatment will be discussed from a clinical perspective as well as a general health perspective. Is loneliness as potentially dangerous as depression?

\section{References:}

Schutter N, Holwerda TJ, Stek ML, Dekker JJM, Rhebergen D, Comijs HC (2017). Loneliness in older adults is associated with diminished cortisol output. Journal of Psychosomatic Research 95: 19-25.

Hegeman A, Schutter N, Comijs HC, Holwerda TJ, Dekker JJM, Stek ML', van der Mast R (2017). Loneliness and cardiovascular disease and the role of late-life depression. International Journal of Geriatric Psychiatry, DOI: 10.1002/gps.4716. 